\title{
MORFOLOGIA DE FRUTOS E SEMENTES DE INHAÍBA (LECYTHIS LURIDA (MIERS) S.A. MORI - LECYTHIDACEAE
}

\author{
Taline Borges Ribeiro ${ }^{1}$, Teresa Aparecida Soares de Freitas ${ }^{2 *}$, Thaine Teixeira Silva ${ }^{3}$
}

\begin{abstract}
RESUMO - O objetivo deste trabalho foi descrever os caracteres morfológicos de frutos e sementes de Lecythis lurida (Miers) S.A. Mori. Os frutos foram coletados no munícipio de Laje - Bahia em abril de 2018. No estudo do fruto, foram observadas as características: tipo, dimensões, textura, consistência do pericarpo e número de sementes por fruto. Os aspectos observados para as sementes foram: externas - cor, forma, dimensões, textura e consistência do tegumento; internas - embrião (cotilédones, eixo hipocótilo-radícula). O fruto de inhaíba é do tipo pixídio, indeiscente, de textura lenhosa, apresentando em média quatro sementes por fruto, com comprimento médio de $5,61 \mathrm{~cm}$ e largura média de $7,48 \mathrm{~cm}$. As sementes apresentam formato triangular anguloso, tegumento ósseo, dotadas de arilo carnoso na região basal e ápice umbonado, com dimensões médias de 43,55 x 36,89 x 29,28 mm. O embrião é conferruminado, sem distinção de cotilédones, eixo hipocótiloradícula e plúmula. A morfologia de frutos e sementes fornece subsídios que contribuem para o reconhecimento da espécie.
\end{abstract}

Palavras chave: descrição morfológica, espécie nativa, sementes florestais.

\section{MORPHOLOGY OF INHAÍBA FRUITS AND SEEDS (LECYTHIS LURIDA (MIERS) S.A. MORI - LECYTHIDACEAE}

\begin{abstract}
The aim of this study was to describe the morphological characters of fruit and seed of Lecythis lurida (Miers) S.A. Mori. The fruits were collected in the municipality of Laje - Bahia in April 2018. In the study of the fruit characteristics were observed: type, dimensions, texture, consistency of the pericarp and number of seeds per fruit. The observed aspects for seeds were: - external: color, shape, dimensions, texture and consistency of the tegument; - internal: embryo (cotyledons, hypocotyl and radicle axis). The fruit of inhaíba is pixídio, indehiscent, woody texture, presenting on average four seeds per fruit, with an average length of $5.61 \mathrm{~cm}$ and average width of $7.48 \mathrm{~cm}$. The seeds are triangular angular shape, bone tegument, endowed with meaty aril in the basal region and umbonado apex, with average size of $43.55 \times 36.89 \times 29.28 \mathrm{~mm}$. The embryo is conferrumined, without distinction of cotyledons, axle hypocotyl and radicle and plumule. The morphology of fruits and seeds provides subsidies that contribute to the recognition of the species.
\end{abstract}

Keywords: forest seeds, morphological description, native species.

\footnotetext{
${ }^{1}$ Engenheira Florestal, br.talyne@gmail.com

${ }^{2}$ Professora do Centro de Ciências Agrárias Ambientais e Biológicas, Campus cruz das Almas - BA, Universidade Federal do Recôncavo da Bahia (CCAAB/UFRB), tas freitas@hotmail.com

${ }^{3}$ Graduanda em Engenharia Florestal da Universidade Federal do Recôncavo da Bahia(CCAAB/UFRB), thaine_17teixeira@hotmail.com
} 


\section{INTRODUÇÃO}

A importância dos estudos morfológicos é decorrente, em grande parte, do fato do seu conhecimento servir como alicerce na identificação das espécies. O estudo da estrutura, fenologia e do comportamento de uma espécie na sua comunidade muitas vezes é restrito devido à falta da sua identidade. Em determinados casos, têm se apenas o fruto, a semente ou a plântula para a sua identificação (Soares et al., 2017).

Associado a caracterização morfológica, as características biométricas de frutos e sementes podem fornecer subsídios importantes para a diferenciação de espécies do mesmo gênero. Além disso, a caracterização biométrica também está relacionada ao modo de dispersão e estabelecimento de plântulas, sendo tal parâmetro utilizado para distinguir espécies pioneiras e não pioneiras em florestas tropicais (Cruz et al., 2001).

Segundo Bewley et al. (2013), as características morfológicas das sementes são diferentes entre classes, famílias e espécies de plantas, com diferenças nos tecidos de reservas e formas de tegumento, mesmo que alguns mecanismos de desenvolvimento das plântulas sejam comuns entre espécies.

Estudos relacionados a aspectos morfológicos de frutos e sementes têm sido realizados para algumas espécies da família Lecythidaceae, tais como: jeniparana (Gustavia augusta L.), castanha-de-sapucaia (Bertholletia excelsa Humboldt \& Bonpland); jequitibárosa (Cariniana legalis (Mart.) Kuntze) e Lecythis pisonis Cambess (Santos et al., 2006; Silva et al., 2014; Ribeiro et al., 2015; Leão et al., 2016).

Lecythis lurida (Miers) S.A. Mori é uma espécie da família Lecythidaceae, conhecida popularmente como jarana, jarana-branca, jarana-buroja, inhaúba, inhaíba ou inhaíba-gigante (Souza et al., 1997). Endêmica do Brasil com ocorrência nos estados do Pará, Bahia, Maranhão, Pernambuco, Piauí, Sergipe, Minas Gerais, Espírito Santo e Rio de Janeiro, e com domínio fitogeográfico na Amazônia e na Mata Atlântica (Flora Brasil, 2019).

A família Lecythidaceae apresenta-se como sendo ecologicamente umas das famílias mais importantes em habitats de terra firme. Em vários estudos de cunho ecológico, evidenciam a importância da família para a manutenção dos ambientes naturais. A distribuição das Lecythidaceae no Novo Mundo ocorre em algumas das áreas que estão sob a maior ameaça de destruição de habitats o que propicia o risco de extinção das espécies de Lecythidaceae e alterações profundas nessas formações vegetais (Mori, 1990). As propriedades da espécie são pouco conhecidas (Taffarel et al., 2014). A madeira de Lecythis lurida é pesada, dura e de alta resistência mecânica e apresenta bom acabamento. Possui uso na construção civil como em vigas, tacos, batentos, embalagens pesadas, carrocerias, cabos de ferramentas e afins (Souza et al., 1997).

Considerando a escassez de estudos referentes à família Lecythidaceae e, em especial, estudos referentes à espécie Lecythis lurida o presente estudo tem por objetivo caracterizar morfologicamente os frutos e sementes desta espécie, visando com isto, fornecer subsídios que irão auxiliar não só no reconhecimento da mesma no campo, como também auxiliar em estudos que visem sua utilização, produção e conservação.

\section{MATERIAL E MÉTODOS}

Os frutos de Lecythis lurida foram colhidos com auxílio de um podão em abril de 2018, em ambientes de pastos com vegetação rasteira no município de Laje, na Bahia. As condições climáticas do município enquadram-se no tipo climático tropical Am da classificação de Koppen, com altitude de 190 metros, nas coordenadas: $13^{\circ} 10^{\prime} 56^{\prime \prime} \mathrm{S}$ e $39^{\circ} 25^{\prime} 30^{\prime \prime} \mathrm{W}$.

Após a colheita, os frutos foram encaminhados ao laboratório de Sementes pertencente à Universidade Federal do Recôncavo da Bahia, em Cruz das Almas - BA, onde foram caracterizados e beneficiados.

Foram coletados aproximadamente 200 frutos, dos quais selecionou-se de forma aleatória 100 frutos dentre eles 100 sementes para descrição dos caracteres morfológicos e biométricos. Nos frutos, foram considerados os seguintes aspectos: tipo, comprimento, largura, textura, consistência do pericarpo e número de sementes por fruto. Nas sementes, as características morfológicas externas observadas e descritas foram: cor, comprimento, largura, espessura, peso, forma, textura e consistência dos tegumentos; e características morfológicas internas, como: embrião (cotilédones, eixo hipocótilo-radícula).

A metodologia e terminologia empregadas, assim como os parâmetros utilizados nas descrições, foram 
baseadas em literaturas de Barroso et al. (2004), Brasil (2009) e Vidal \& Vidal (2013).

As análises biométricas dos frutos (comprimento e largura) foram obtidas com régua milimetrada e das sementes (comprimento, largura e espessura) foram obtidas com paquímetro digital com precisão de 0,01 $\mathrm{mm}$ e, a massa individual das sementes foi determinada por meio de uma balança de precisão $(0,0001 \mathrm{~g})$.

Os dados foram submetidos à análise descritiva, com cálculos de média aritmética, máximo, mínimo, coeficiente de variação e erro padrão da média. As ilustrações foram feitas manualmente, selecionandose materiais sadios e representativos como modelos.

\section{RESULTADOS E DISCUSSÃO}

A espécie apresenta fruto do tipo pixídio, globoso, grande, simples, seco, lenhoso, polispérmico e indeiscente. $\mathrm{O}$ fruto se divide em duas porções distintas: urna (inferior) e opérculo (superior). As estruturas do fruto de Lecythis lurida podem ser visualizadas na Figura 1. O ápice é umbonado e, na região de inserção do pedúnculo, ocorre liberação de exsudato (Figura 1A). Em corte longitudinal o fruto apresenta formato oval (Figura 1C).

Semelhante ao observado em L. lurida, a espécie Gustavia augusta L., também da família Lecythidaceae, apresenta fruto do tipo pixídio indeiscente, conforme observado por Silva et al. (2014).
O pericarpo é lenhoso, globoso e rígido, de coloração marrom quando maduro. Exocarpo marrom, opaco, reticulado, com lenticelas estouradas, glabro, fibroso. O endocarpo é divido em lóculos, nos quais as sementes são distribuídas, podendo conter entre 2 a 11 sementes, com média de 4 sementes por fruto, havendo mais sementes por lóculo quando as sementes são menores (Figura 2).

Em estudo realizado por Tsou \& Mori (2002) observaram que o arilo da semente de Gustavia augusta L. (Lecythidaceae) são expostos, por apodrecimento do fruto e, com isso ocorre a atração de animais, que se alimentam e dispersam as sementes. Portanto, as características dos frutos permitem a dispersão zoocórica, realizada principalmente por roedores e pequenos mamíferos e, em alguns casos, por peixes.

Informações sobre o número de sementes por fruto são relevantes para o planejamento de coleta de sementes e em testes de germinação. Dispondo deste dado, o coletor poderá planejar o quanto, em média, necessitará coletar de frutos para obter determinado número de sementes. Entretanto, tal informação não deve ser utilizada como indicativo de produção, visto que populações e indivíduos da mesma espécie podem apresentar diferentes resultados (Ferreira et al., 2001).

As determinações biométricas dos frutos de $L$. lurida encontram-se na Tabela 1 . O comprimento variou de 3,80 a $7,20 \mathrm{~cm}$, enquanto a largura apresentou de 4,80 a $9,60 \mathrm{~cm}$.
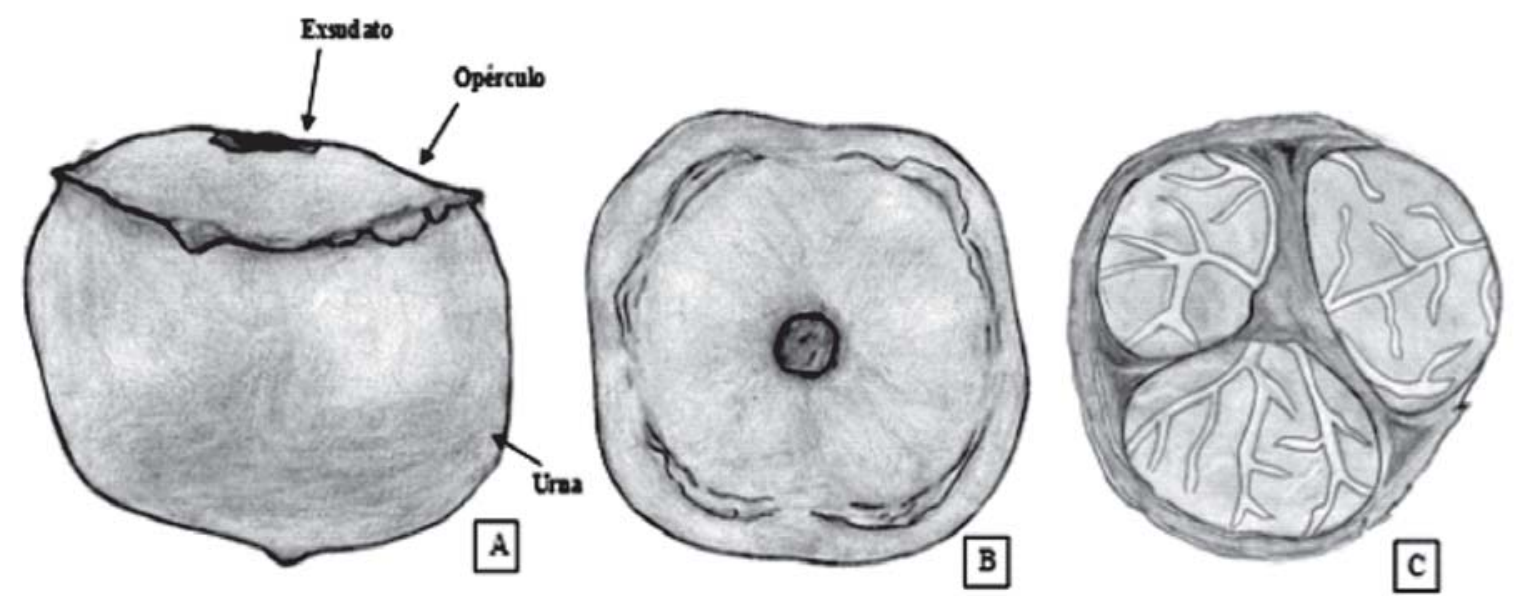

Figura 1 - A-B: Estrutura de um fruto de L. lurida. A=Fruto fechado, em posição longitudinal; $\mathrm{B}=$ Fruto observado em posição transversal; $\mathrm{C}=$ Fruto em corte transversal, sem opérculo, ainda com as sementes no interior da urna. 


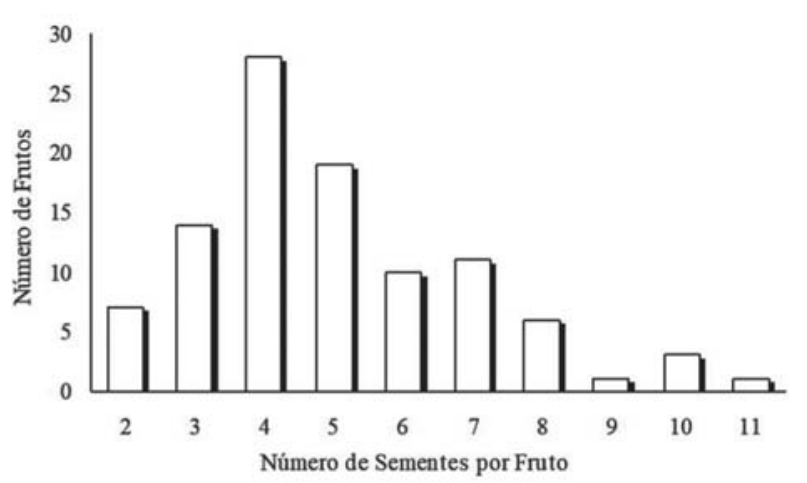

Figura 2 - Distribuição do número de sementes por fruto de Lecythis lurida.

Tabela 1 - Média, máximo, mínimo, erro padrão da média e coeficiente de variação, referentes às determinações biométricas em uma amostra de 100 frutos de Lecythis lurida.

\begin{tabular}{lcc}
\hline \multicolumn{3}{c}{ Frutos } \\
\hline Parâmetros & Comprimento $(\mathrm{cm})$ & Largura $(\mathrm{cm})$ \\
\hline Média & 5,61 & 7,48 \\
Máximo & 7,20 & 9,60 \\
Mínimo & 3,80 & 4,80 \\
EPM & 0,08 & 0,10 \\
CV (\%) & 14,41 & 13,45 \\
\hline
\end{tabular}

EPM= Erro padrão da média; $C V=$ Coeficiente de Variação.

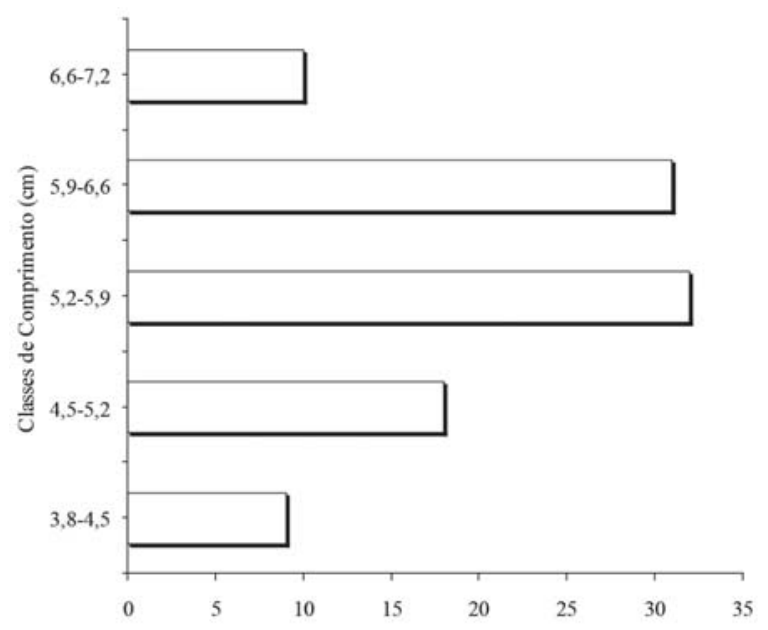

Os frutos possuem comprimento médio de 5,61 $\mathrm{cm}$, com predominância de frutos com comprimento entre 5,2 a 5,9 cm; largura média de 7,48 cm e predominância entre 6,7 a 7,7 cm (Figura 3A-B).

As sementes apresentam formato triangular anguloso, são assimétricas nos lados, rugosas e dotadas de arilo vestigial carnoso na região basal. Beltrati (1994) define arilo como excrescência do hilo ou do funículo, no entanto, Brasil (2009) usa o termo para qualquer excrescência carnosa da semente. Segundo Barroso et al. (2004), esta estrutura é visualizada em diversas sementes do gênero Lecythidaceae, como encontrado para Eschweilera ovata, Cariniana legalis e Lecythis pisonis por Oliveira et al. (2012), Ribeiro et al. (2015) e Leão et al. (2016), respectivamente.

O tegumento apresenta textura lenhosa e óssea, sendo a testa de coloração marrom-escura a marromclaro, rugosa, opaca e com presença de nervuras de coloração castanho-claro (Figura 4A).

O embrião apresenta-se conferruminado, atípico, com estruturas ovóides, elipsóides e globosas, não havendo distinção de cotilédones, eixo hipocótiloradícula e plúmula (Figura 5).

De acordo com Barroso et al. (2004), espécies do gênero Lecythidaceae, podem apresentar o embrião sem distinção entre suas estruturas. Porém os mesmos autores citam que espécies pertencentes a esse gênero também podem apresentar cotilédones bem constituídos

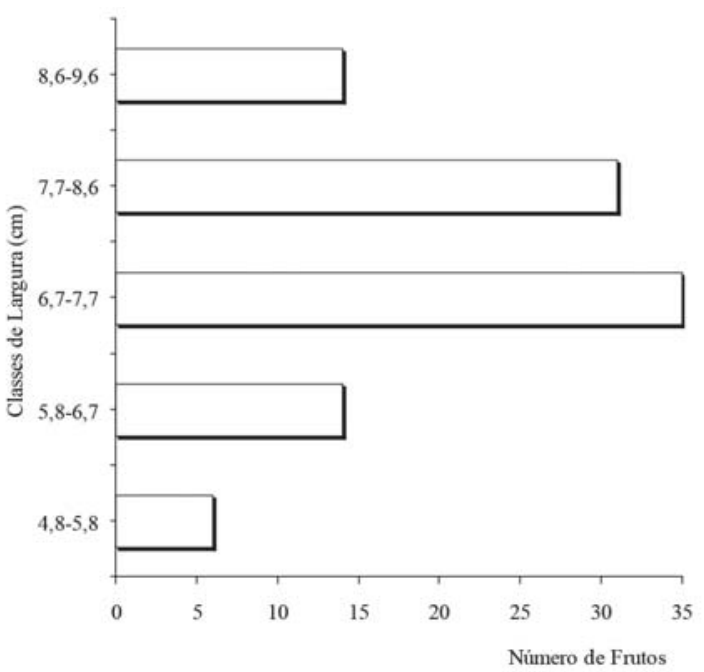

Figura 3 - Distribuição das classes de comprimento $(\mathrm{cm})$ e largura $(\mathrm{cm})$ para uma amostra de 100 frutos de inhaíba (Lecythis lurida). 

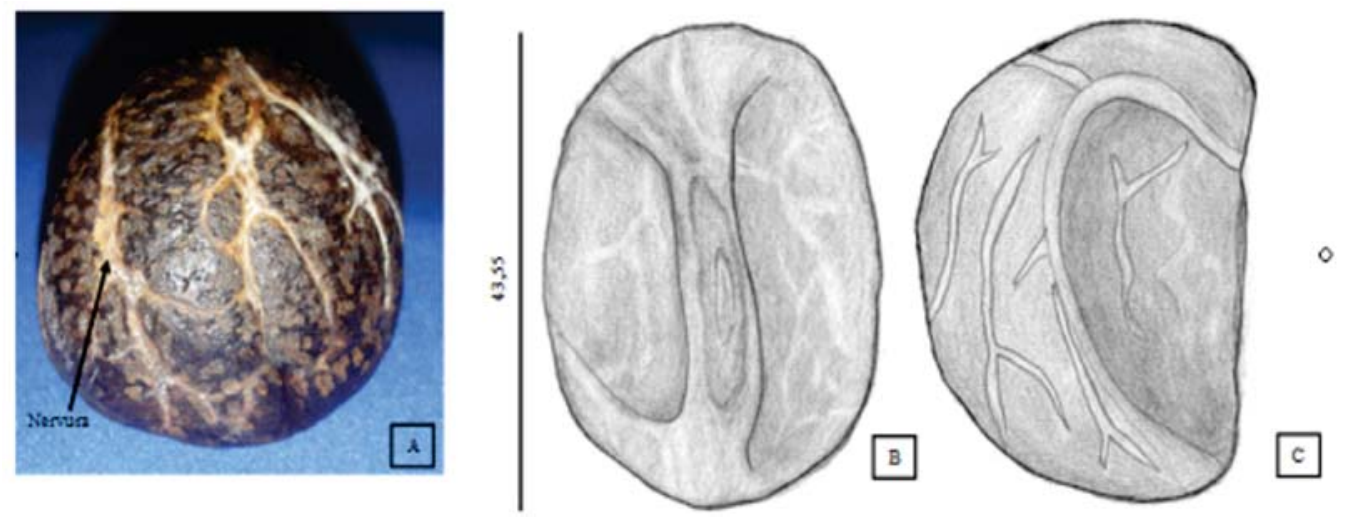

Figura 4 - Estrutura da semente de Lecythis lurida: A- Semente intacta; B-C: Esquematização da semente em ângulos diferentes.

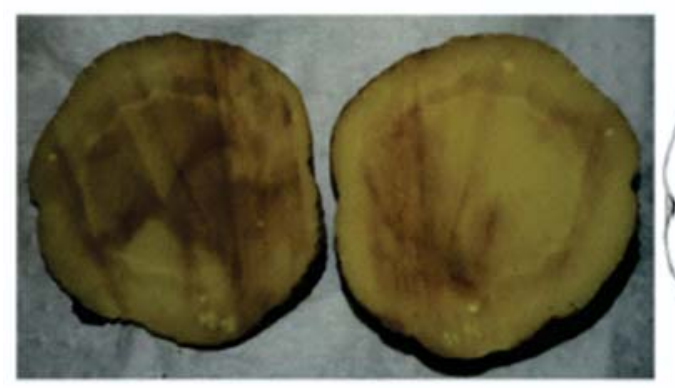

A

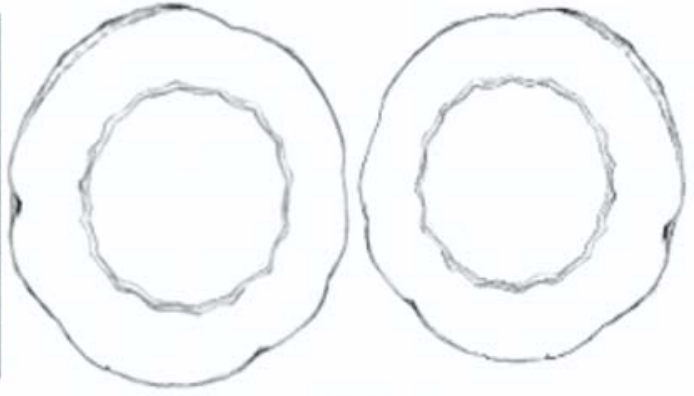

B

Figura 5 - A: Semente de Lecythis lurida em corte longitudinal. B: Esquematização da semente de Lecythis lurida em corte longitudinal.

e eixo hipocótilo-radícula distintos, como observado para Cariniana legalis (Mart.) Kuntze por Ribeiro et al. (2015). Estes autores relatam que a variação do embrião em espécies de Lecythidaceae está relacionada a estratégias de crescimento e estabelecimento de plântulas.

As determinações biométricas das sementes de L. lurida encontram-se na Tabela 2. O comprimento variou de 29,39 a 58,08 mm, enquanto a largura e espessura apresentaram valores de 19,58 a $58,43 \mathrm{~mm}$ e 17,60 a $47,98 \mathrm{~mm}$, respectivamente. Para o peso das sementes foram encontrados valores de 6,16 a 56,69 g por unidade/ semente.

De acordo com os resultados pode-se constatar que as sementes de inhaíba apresentam elevada variação em todas as dimensões avaliadas, gerando alto coeficiente de variação. Segundo Leão et al. (2016), elevados coeficientes de variação podem ser decorrentes das condições genéticas das árvores matrizes e da interação dessas com diferentes condições edáficas e climáticas.

Em relação às frequências das dimensões mensuradas nas sementes de inhaíba, verificou-se que a maior parte das sementes possui de 35,1 a $40,9 \mathrm{~mm}$ de comprimento, 35,1 a 42,9 mm de largura, 17,6 a 23,7 $\mathrm{mm}$ de espessura e 6,2 a 16,3 gramas por unidade de semente (Figura 6A-D). Valores similares para tais dimensões foram encontrados para Lecythis pisonis cambess por Braga et al. (2007).

A média do comprimento foi de $43,55 \mathrm{~mm}$, caracterizando a semente como grande. O tamanho de uma semente influencia no estabelecimento da espécie e sua dispersão, sendo relacionado com a competição, predação e distribuição no espaço. Sementes de tamanhos 
maiores possuem menor restrição em condições naturais, o que lhe atribui vantagens adaptativas (Lusk \& Kelly, 2003).

\section{CONCLUSÃO}

Lecythis lurida apresenta frutos do tipo pixídio, indeiscentes e globosos. As sementes são constituídas de um arilo vestigial carnoso na região basal e embrião conferruminado. As dimensões de comprimento, largura, espessura e a massa das sementes são bastante variáveis.
A descrição morfológica do fruto e da semente realizada neste trabalho poderá ser utilizada em estudos de regeneração em ambientes de ocorrência natural de L. lurida, auxiliando também na identificação desta espécie em banco de sementes.

No entanto, faz-se necessário também a realização de um estudo referente a germinação e a morfologia da plântula desta espécie, contribuindo para melhor conhecimento do seu ciclo biológico.

Tabela 2 - Média, máximo, mínimo, erro padrão da média e coeficiente de variação, referentes às determinações biométricas em uma amostra de 100 sementes de Lecythis lurida

\begin{tabular}{lcccc}
\hline \multicolumn{3}{c}{ Sementes } & & \\
\hline Parâmetros & Comprimento $(\mathrm{mm})$ & Largura $(\mathrm{mm})$ & Espessura(mm) & Peso(g) \\
\hline Média & 43,55 & 36,89 & 29,28 & 27,42 \\
Máximo & 58,08 & 58,43 & 47,98 & 56,69 \\
Mínimo & 29,39 & 19,58 & 17,60 & 6,16 \\
EPM & 0,08 & 0,85 & 0,70 & 1,33 \\
CV $(\%)$ & 18,34 & 22,95 & 23,98 & 48,48 \\
\hline
\end{tabular}

$\mathrm{EPM}=$ Erro padrão da média; $\mathrm{CV}=$ Coeficiente de variação.
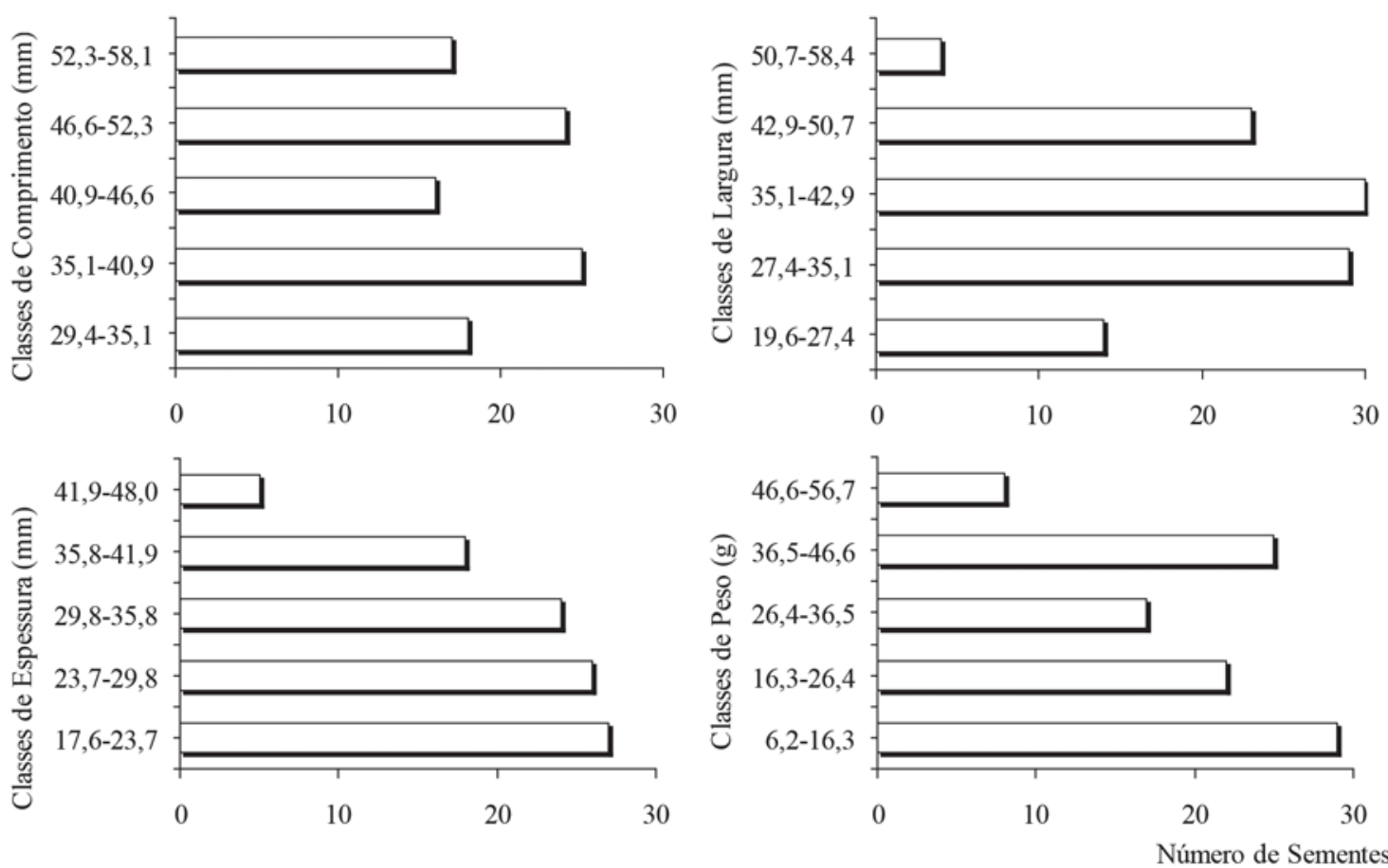

Figura 6 - Distribuição das classes de comprimento, largura, espessura e peso para sementes de inhaíba (Lecythis lurida). 


\section{LITERATURA CITADA}

BARROSO, G.M.; MORIN, M.P.; PEIXOTO, A.L. et al. Frutos e sementes. Edição: 1. Editora: Universidade Federal de Viçosa, 2004.

BELTRATI, C.M. Morfologia e anatomia de sementes. Rio Claro: UNESP, 1994.

BEWLEY, J.D.; BRADFORD, K.; HILHORST, H. Seeds: physiology of development, germination and dormancy. 3.ed. New York: Springer, 2013.

BRAGA, L.F.; SOUZA, M.P. ; GILBERTI, S. et al. Caracterização morfométrica de sementes de castanha de sapucaia (Lecythis pisonis cambess Lecythidaceae). Revista de Ciências AgroAmbientais, Alta Floresta, v.5, n.1, p.111-116, 2007.

BRASIL. Ministério da Agricultura, Pecuária e Abastecimento. Glossário ilustrado de morfologia / Ministério da Agricultura, Pecuária e Abastecimento. Secretaria de Defesa Agropecuária. Brasília: Mapa/ACS, 2009.

CRUZ, E.D.; MARTINS, F.O.; CARVALHO, J.E.U. Biometria de frutos e sementes e germinação de jatobá-curuba (Hymenaea intermedia Ducke, Leguminosae - Caesalpinioideae). Revista Brasileira de Botânica, v.24, n.2, p.161-165, 2001.

FERREIRA, R.A.; BOTELHO, S.A.; DAVIDE, A.C.; MALAVASI, M.M. Morfologia de frutos, sementes, plântulas e plantas jovens de Dimorphandra mollis Benth.- faveira (Leguminosae - Caesalpinioideae). Revista Brasileira de Botânica, v.24, n.3, p.303-309, 2001.

LEÃO, N.V.M.; ARAÚJO, E.A.A.; SHIMIZU, E.S.C. et al. Características biométricas e massa de frutos e sementes de Lecythis pisonis cambess. Enciclopédia Biosfera, Centro Científico Conhecer, v.13, n.24, p.167-175, 2016.

MORI, S. Diversificação e conservação das Lecythidaceae neotropicais. Acta Botanica Brasilica, Feira de Santana, v.4, n.1, p.4568, July 1990. Available from <http://www.scielo.br/ scielo.php?script $=$ sci_arttext\&pid $=\mathrm{S} 0102$ $33061990000100004 \& \operatorname{lng}=$ en\&nrm $=$ iso $>$. access on 15 June 2019. http://dx.doi.org/10.1590/S010233061990000100004.
FLORA BRASIL 2020. Lecythidaceae in Flora do Brasil 2020 em construção. Jardim Botânico do Rio de Janeiro. Disponível em: $<$ http://reflora.jbrj.gov.br/reflora/floradobrasil/ FB8560>. Acesso em: 17 Jun. 2018.

LUSK, C.H.; KELLY, C.K. Interspecific variation in seed size and safe sites in a temperate rain forest. New Phitologist, v.158, p.535-541, 2003.

OLIVEIRA, R.G.; MATOS, V.P.; MONTEIRO, H.A. et al. Morfologia do fruto, semente e plântula de Eschweilera ovata (Cambess.) Miers. Ciência Florestal, v.22, n.2, p.371-377, 2012.

RIBEIRO, R.T.M.; SILVA, R.M.; GALLAO, M. Morfologia de frutos e sementes de Cariniana legalis (Mart.) Kuntze (Lecythidaceae). Scientia Amazonia, v.4, n.2, 2015. SANTOS, J.U.M.; BASTOS, M.N.C.; GURGEL, E.S.C. et al. Bertholletia excelsa Humboldt \& Bonpland (Lecythidaceae): aspectos morfológicos do fruto, da semente e da plântula. Bol. Mus. Para. Emílio Goeldi. Ciências Naturais, v.1, n.2, p.103-112, 2006.

SILVA, R.M.; RIBEIRO, R.T.M.; COUTINHO, D.J.G. et al. Caracterização de frutos, sementes, plântulas e germinação de Jeniparana. Revista Ceres, v.61, n.5, p.746-751, 2014.

SOARES, I.D.; NOGUEIRA, A.C.; GRABIAS, J. et al. Caracterização morfológica de fruto, semente e plântula de Psidium rufum DC. (Myrtaceae). Iheringia, Série Botânica, v.72, n.2, p.221-227, 2017.

SOUZA, M.H.; MAGLIANO, M.M.; CAMARGOS, J.A.A. Madeiras Tropicais Brasileiras. Brasília, DF: IBAMA. 1997.

TAFFAREL, M.; CARVALHO, J.O.P.; MELO, L.O. et al. Efeito da silvicultura pós-colheita na população de Lecythis lurida (Miers) Mori em uma floresta de terra firme na Amazônia Brasileira. Ciência Florestal, v.24, n.4, p.889898, 2014. 
TSOU, C.; MORI, S.A. Seed coat anatomy and its relationship to seed dispersal in subfamily Lecythidoideae of the Lecythidaceae (The Brazil Nut Family). Botanical Bullentin Academia Sinica, v.43, p.37-56, 2002.
VIDAL, W.N.; VIDAL, M.R.R. Botânicaorganografia: quadros sinóticos ilustrados de fanerógamos. UFV, Viçosa, Brasil. $4^{\circ}$ Edição. 2013. $124 \mathrm{p}$.

Recebido para publicação em 29/03/2019 e aprovado em 26/06/2019. 